\title{
Invasão biológica de Artocarpus heterophyllus Lam. (Moraceae) em um fragmento de Mata Atlântica no Nordeste do Brasil: impactos sobre a fitodiversidade e os solos dos sítios invadidos
}

Juliano Ricardo Fabricante ${ }^{1,5}$, Kelianne Carolina Targino de Araújo ${ }^{2}$, Leonaldo Alves de Andrade ${ }^{3}$ e Jéssica Viviane Amorim Ferreira ${ }^{4}$

Recebido em 28/06/2011. Aceito em 27/02/2012

\begin{abstract}
RESUMO
(Invasão biológica de Artocarpus heterophyllus Lam. (Moraceae) em um fragmento de Mata Atlântica no Nordeste do Brasil: impactos sobre a fitodiversidade e solos dos sítios invadidos). O objetivo do trabalho foi avaliar os impactos causados por A. heterophyllus sobre a riqueza e diversidade da vegetação e sobre os solos de um fragmento de Floresta Ombrófila Aberta, Município de Areia, PB. A área estudada localiza-se no Campus II da Universidade Federal da Paraíba. No fragmento, foram instaladas 10 pares de parcelas de $100 \mathrm{~m}^{2}$. Metade destas unidades amostrais (AI ambiente invadido) foi disposta de forma que apresentasse em seu centro um exemplar adulto de A. heterophyllus. Paralelamente a cada uma destas parcelas, outra unidade amostral (AN - ambiente natural) foi alocada. Foi avaliado o número de espécies, a abundância de indivíduos, a diversidade e a equabilidade, além da química dos solos. Foram amostrados 14.008 indivíduos pertencentes a 79 espécies, dos quais 12.369 indivíduos de 50 espécies no AI e 1.639 indivíduos de 75 espécies no AN. A diversidade e a equabilidade geral foram, respectivamente, 0,24 e 0,06 no AI e 3,42 e 0,79 no AN. Todas as variáveis apresentaram diferenças significativas demonstrando que A. heterophyllus modifica os ambientes onde se dispersa. Quanto aos solos, alguns elementos tiveram suas concentrações mudadas enquanto outros não. Constatou-se que A. heterophylus altera de forma contundente a riqueza de espécies, a diversidade da vegetação e os solos dos sítios invadidos, o que torna necessárias ações de mitigação para o controle da mesma.
\end{abstract}

Palavras-chave: bioinvasão, Floresta Ombrófila Aberta, jaca

\begin{abstract}
(Biological invasion of Artocarpus heterophyllus Lam. (Moraceae) in an Atlantic Forest fragment in Northeastern Brazil: impacts on phytodiversity and soils of invaded sites). The objective of this study was to evaluate the impacts caused by A. heterophyllus on the richness and diversity of vegetation, and the soils, in a fragment of open rainforest, in Areia, PB. The study area is located in the Campus II of Universidade Federal da Paraíba. In this fragment, 10 pairs of plots of $100 \mathrm{~m}^{2}$ were established. Half of these sampling units (AI - environment invaded) were laid out so there was an adult $A$. heterophyllus in the center. Alongside each of these plots, one sample unit (AN - natural environment) was allocated. We evaluated the number of species, the abundance of individuals, diversity and evenness, and the chemistry of the soils. We sampled 14,008 individuals belonging to 79 species, of which there were 12,369 individuals and 50 species in the AI and 1,639 individuals and 75 species in the AN. The diversity and evenness of AI were 0.24 and 0.06 , respectively, and for AN they were 3.42 and 0.79 . All variables were significantly different showing that $A$. heterophyllus modify the environments where it grows. The concentrations of some elements in the soil changed while others did not. It was found that $A$. heterophylus forcefully alters species richness, diversity of vegetation and soils of invaded sites, which suggests that actions to control this species are necessary.
\end{abstract}

Key words: bioinvasion, jackfruit, Rain Forest

\footnotetext{
Universidade Federal do Vale do São Francisco, Centro de Referência para Recuperação de Áreas Degradadas, Petrolina, PE, Brasil

2 Universidade Estadual da Paraíba, Graduação em Ciências Biológicas, Campina Grande, PB, Brasil

3 Universidade Federal da Paraíba, Departamento de Fitotecnia, Areia, PB, Brasil

4 Universidade Federal do Vale do São Francisco, Graduação em Ciências Biológicas, Petrolina, PE, Brasil

5 Autor para correspondência: julianofabricante@hotmail.com
} 


\section{Introdução}

O domínio da Mata Atlântica abrange 15 Estados brasileiros e possui área original de ca. $1.300 .000 \mathrm{~km}^{2}$ (SNE 2002, MMA 2004) que se estende do Cabo de São Roque a 5 S , no Estado do Rio Grande do Norte até o Rio Taquari a $30^{\circ} \mathrm{S}$ no Estado do Rio Grande do Sul (Joly et al. 1999). Estima-se que restam atualmente apenas 11-16\% de sua cobertura original (Ribeiro et al. 2009). Em particular, o Estado da Paraíba que originalmente possuía $12 \%$ de seu território coberto por essas formações florestais, teve sua área drasticamente reduzida para 1,2\%, ou seja, um decréscimo na ordem de 90,3\% (SNE 2002). Tida como a $2^{\text {a }}$ floresta mais ameaçada do planeta e enquadrada entre os Hotspots pela Organização Conservation International (2011), a Mata Atlântica possui em seus domínios mais de 15.700 espécies de plantas das quais por volta de 7.100 apresentam algum grau de endemismo (Stehmann et al. 2009).

Atualmente, a diversidade da Mata Atlântica se encontra bastante ameaçada, devido à intensa ocupação antrópica sofrida ao longo do tempo. A situação da região Nordeste é ainda mais grave por possuir um grau de degradação maior que em outras regiões do Brasil (Silva \& Tabarelli 2000), em decorrência da devastação de grandes áreas para a implantação da monocultura da cana-de-açúcar, que resultou no surgimento de uma paisagem extremamente fragmentada (Silva et al. 2006). A antropização sofrida pela Mata Atlântica facilita a ocorrência de um fenômeno ameaçador à sua biodiversidade, que são as invasões biológicas. De acordo com Ziller \& Galvão (2002), invasão biológica é o processo de introdução e consequente adaptação de espécies que não fazem parte naturalmente de um determinado ecossistema, mas que se naturalizam e passam a provocar mudanças em seus processos ecológicos.

Uma das espécies que vem demonstrando comportamento invasor na Mata Atlântica é Artocarpus heterophyllus (jaca ou jaqueira). Árvore, pertencente à família Moraceae, nativa do sudeste asiático (Chaves et al. 1967, Ferrão 1993), que devido aos seus frutos carnosos, foi introduzida em diversos países com o intuito alimentar, porém, tem se tornado indesejável em alguns locais, a exemplo do Parque Nacional da Tijuca no Rio de Janeiro (Abreu \& Rodrigues 2010). Naquele Estado, a espécie foi introduzida a aproximadamente 150 anos por meio de um reflorestamento (Bandeira 1993).

Estes relatos também são corroborados por observações in loco no Campus II da Universidade Federal da Paraíba (UFPB), no município de Areia e se repetem em vários fragmentos da região dos "brejos de altitude" (disjunção de Mata Atlântica) e na Mata Atlântica do litoral nordestino (J. R. Fabricante, dados não public.). As populações da espécie em questão vêm ocorrendo ora em ambientes antropogênicos, ora em meio à vegetação nativa, principalmente em suas bordas, porém não restrita às mesmas. Neste contexto, o presente trabalho busca primeiramente responder se $A$. heterophyllus altera de maneira significativa aspectos biofísicos do fragmento em questão.

Em adição, a área potencial de invasão da espécie foi aqui determinada por meio de modelagem de nicho ecológico, visando subsidiar inferências sobre a amplitude do problema no Brasil. A modelagem de nicho é uma ferramenta que vem sendo amplamente utilizada, principalmente, para a tomada de decisão em iniciativas conservacionistas (Elith et al. 2006) e em estudos com espécies exóticas, é um importante instrumento para o desenvolvimento de estratégias de manejo dos ambientes susceptíveis à invasão das espécies (Guglieri et al. 2009).

Especificamente, os objetivos do trabalho foram avaliar os impactos causados por A. heterophyllus sobre a riqueza, diversidade da fitocenose e solos de um fragmento de Floresta Ombrófila Aberta e avaliar a susceptibilidade das formações vegetacionais brasileiras à invasão por esta espécie. A obtenção desses dados visa diagnosticar a situação da espécie em uma formação florestal nordestina e subsidiar políticas públicas adequadas para o manejo da espécie.

\section{Material e métodos}

A área estudada localiza-se no Centro de Ciências Agrárias, Campus II da UFPB, município de Areia, PB. O estudo foi conduzido em um fragmento de Floresta Ombrófila Aberta, com área de um pouco mais de 50 ha $\left(6^{\circ} 58^{\prime} 1,9^{\prime \prime}\right.$ S e $35^{\circ} 43^{\prime} 4,2^{\prime \prime}$ W, $592 \mathrm{~m}$ de altitude média). Segundo moradores antigos da vizinhança, a vegetação do fragmento nunca foi totalmente suprimida, mas sempre sofreu corte seletivo e com a instalação da Universidade, essa prática foi reduzida consideravelmente.

O local está situado sob o clima do tipo As', segundo a classificação de Koppen (McKnight \& Hess 2000), ou seja, tropical quente úmido, com precipitação media anual superior a $1.400 \mathrm{~mm}$. Os solos são predominantemente Podzólicos Vermelho-Amarelo Equivalente Eutrófico (Brasil 1972).

Foram instaladas 10 pares de parcelas de $100 \mathrm{~m}^{2}(10 \mathrm{x} 10$ $\mathrm{m})$. Metade destas unidades amostrais (AI - ambiente invadido) foi disposta de forma que apresentasse em seu centro um indivíduo adulto de $A$. heterophyllus. Paralelamente a cada uma dessas parcelas, a uma distância máxima de $2 \mathrm{~m}$ e mínima de $1 \mathrm{~m}$, outra unidade amostral (AN - ambiente natural) foi alocada. Estas foram dispostas em sítios típicos do fragmento, ou seja, não invadidos pela espécie em questão, sendo considerados, portanto, como testemunhas para o teste da hipótese do presente estudo.

No interior das parcelas todos os indivíduos arbustivos e arbóreos foram contabilizados e com esses valores foi calculada a densidade (Dens) das espécies (Müller-Dombois \& Ellemberg 1974). Também foi avaliada a diversidade segundo o índice de Diversidade de Shannon-Weaver $\left(H^{\prime}\right)$ (Shannon \& Weaver 1949), e a equabilidade, através do índice de Pielou (E) (Pielou 1977).

O número de indivíduos e o número de espécies foram comparados por meio do teste de Kruskal-Wallis $(\mathrm{H})(p$ 
$\leq 0,05)$, com as diferenças entre os postos avaliada por Dunn $(p \leq 0,05)$ (Zar 1999), quando os dados apresentaram distribuição não-normal ou, por Tukey $(p \leq 0,05)$ (Tukey 1959), quando a distribuição era normal. A normalidade dos valores foi avaliada por D’Agostino $(p \leq 0,05)$ (D'Agostino et al. 1990). Diferenças entre as diversidades foram verificadas pelo teste $\mathrm{t}(p \leq 0,05)$ (Lehmann 1997). Para avaliar a similaridade entre os ambientes foram utilizados o coeficiente binário de Jaccard (Sj) (Müller-Dombois \& Ellemberg 1974) e o de distância de Bray-Curtis $\left(\mathrm{D}_{\mathrm{A}-\mathrm{B}}\right)$ (Bray \& Curtis 1957).

Em cada parcela quatro amostras de solo na profundidade de $0-20 \mathrm{~cm}$ foram coletadas e homogeneizadas para compor uma amostra composta por unidade amostral. Com elas foram realizadas análises químicas seguindo os procedimentos recomendados por EMBRAPA (1997). Os resultados foram submetidos a analise de variância, com as médias dos ambientes comparadas pelo teste Tukey ( $p \leq$ 0,05) (Tukey 1959).

Visando inferir sobre a área potencial de dispersão/ invasão de A. heterophyllus no Brasil, foi realizada uma análise de modelagem de nicho ecológico. Os pontos de georreferenciamento de ocorrência da espécie foram obtidos em trabalhos científicos (Nunes 2004, Abreu \& Rodrigues 2005, Abreu 2008, Boni et al. 2009, Abreu \& Rodrigues 2010, Novelli et al. 2010) e nas bases de dados do CRIA (2011) e do Instituto Horus (2011). Para a geração do modelo preditivo de hábitat potencial, foi utilizado o algoritmo BIOCLIM, que é considerado um dos mais eficientes para este tipo de análise em regiões da América do Sul (Elith et al. 2006).

A classificação taxonômica foi elaborada de acordo com o Sistema APG III (2009), disponível na base de dados Tropicos (2010) do Missouri Botanical Garden, Saint Louis, Missouri, USA. As análises estatísticas foram executadas utilizando-se os softwares Past $1.12^{\circ}$ (Hammer et al. 2003), MVSP $3.1^{\oplus}$ (Kovach 2005), BioEstat $5.0^{\odot}$ (Ayres et al. 2007) e DIVA-GIS $5.2^{\circ}$ (Hijmans et al. 2009).

\section{Resultados e discussão}

Foram amostrados 14.008 indivíduos $($ Dens $=70.040$ indivíduos/ha) pertencentes a 79 espécies, dos quais 12.369 indivíduos (Dens $=123.690$ indivíduos/ha) de 50 espécies no AI e 1.639 indivíduos (Dens = 16.390 indivíduos/ha) de 75 espécies no AN. Do total de indivíduos do AI, 11.955 (Dens $=119.550$ indivíduos/ha) eram de A. heterophyllus e 414 (Dens $=4.140$ indivíduos/ha) eram das outras 49 espécies (Tab. 1).

A alta densidade observada para A. heterophyllus no fragmento estudado é diferente dos padrões de abundância da espécie em sua área de dispersão original. Na Índia ela é considerada rara, apresentando poucos indivíduos por hectare (Chittibabu \& Parthasarathy 2000). Alguns fatores apontados por Abreu (2008), tais como (i) diferenças climáticas entre os locais, como ausência de uma estação seca marcante, característica das regiões de clima de monção, (ii) ausência de espécies que dominem os estratos da vegetação, assim como ocorre em Florestas Tropicais asiáticas, (iii) produção elevada de frutos, e (iv) alto índice de germinação das sementes em campo, seriam prováveis facilitadores para a explosão populacional verificada.

Os valores da densidade de A. heterophyllus, porém, devem ser relativizados uma vez que as parcelas do AI foram direcionadas para onde haviam matrizes da espécie. Se calcularmos a densidade da exótica tomando como área amostral os 50 ha do fragmento, a mesma possuiria 2.391 indivíduos/ha. A despeito de bem menor, essa abundância ainda se diferencia enormemente da encontrada nas suas áreas de ocorrência natural e é maior que das espécies autóctones encontradas em estudos realizados nas formações florestais da região (Oliveira et al. 2006, Xavier et al. 2009). Nas parcelas do AN as duas espécies com maiores densidades também apresentaram valores inferiores: 1.930 indivíduos ha (Erythroxylum simonis) e 1.580 indivíduos ha (Brosimum guianense). O número de espécies no AI foi de $14,7 \pm 7,9$ e no AN 25,9 $\pm 8,8$ (média \pm desvio padrão, em todo texto). A diferença observada foi significativa (teste de Tukey, $\mathrm{F}=8,95, \mathrm{p}=0,0077$ ), demonstrando que a riqueza de espécies é menor onde há a presença de $A$. heterophyllus (Fig. 1a).

$\mathrm{O}$ número de indivíduos, quando se inclui $A$. heterophyllus na avaliação, foi de $1.236,9 \pm 842,2$ no AI e de 163,9 $\pm 86,9$ no AN, e de $41,4 \pm 31,5$ indivíduos quando se exclui A. heterophyllus. As diferenças na abundância de espécimes entre os ambientes foram significativas para ambos os casos, sendo que com a presença de $A$. heterophyllus ela foi maior no $\mathrm{AI}(\mathrm{H}=13,17, \mathrm{p}=0,0003)$ e com a exclusão da espécie, foi maior no $\mathrm{AN}(\mathrm{F}=14,16, \mathrm{p}=0,0017)$ (Fig. 1b e 1c).

Fatores tais como a existência de substâncias alelopáticas presentes nos tecidos do táxon (Perdomo \& Magalhães 2007) e a alteração da química e fertilidade dos solos ocasionada pela presença da espécie (ver abaixo), concorrem para explicar as mudanças constatadas. Exclusão de espécies, aumento na densidade por área e diminuição do número de indivíduos das espécies nativas são apontados por diversos autores como as principais consequências da invasão biológica (e.g., Breytenbach 1986, Versfeld \& Van Wilgen 1986, Rapoport 1991, Richardson \& Higgins 1998, Parker et al. 1999, Rejmánek et al. 2005, Ziller \& Zalba 2007, Andrade et al. 2008, Andrade et al. 2009, Andrade et al. 2010, Abreu \& Rodrigues 2010).

Quase todas as parcelas apresentaram similaridade, não havendo uma diferenciação entre aquelas invadidas e as não invadidas pela análise de Jaccard (Fig. 2). Porém, as parcelas no AI e no AN apresentaram dissimilaridade entre si, formando dois grupos distintos quando os dados foram submetidos à análise de Bray-Curtis (Fig. 3). A diferença nesses resultados demonstra que os impactos da invasão de A. heterophyllus sobre a fitocenose são mais evidenciados quando se considera não só a presença e ausência de espécies, mas, também a abundância das espécies. 
Tabela 1. Lista de espécies amostradas por ambiente em um fragmento de Mata Atlântica, Areia, PB. Sendo: AI - Ambiente Invadido; AN - Ambiente Natural.

\begin{tabular}{|c|c|c|}
\hline Família/Espécie & Nome Vulgar & Ambiente \\
\hline \multicolumn{3}{|l|}{ ANACARDIACEAE } \\
\hline Mangifera indica $\mathrm{L}$. & Manga & AI e NA \\
\hline Spondias mombin L. & Cajá & AI e NA \\
\hline Tapirira guianensis Aubl. & Cupiúba Branca & AI e NA \\
\hline Tapirira sp1. & Cupiúba Preta & NA \\
\hline Thyrsodium spruceanum Salzm. ex Benth. & Caboatam Rosa & AI e NA \\
\hline sp1. & Cupiúba Rosa & AI e NA \\
\hline \multicolumn{3}{|l|}{ ANNONACEAE } \\
\hline Guatteria sp. & Maria Preta & NA \\
\hline \multicolumn{3}{|l|}{ APOCYNACEAE } \\
\hline Himatanthus phagedaenicus (Mart.) Woodson. & Lagarteiro & AI e NA \\
\hline Tabernaemontana heterophilla Vahl. & Leiteiro & AI e NA \\
\hline \multicolumn{3}{|l|}{ ARACACEAE } \\
\hline Acrocomia intumescens Drude & Macaíba & $\mathrm{AI}$ e NA \\
\hline Attalea oleifera Barb. Rodr. & Pindoba & NA \\
\hline \multicolumn{3}{|l|}{ ARALIACEAE } \\
\hline Schefflera morototoni (Aubl.) Steyemark \& Frodin & Sabaquim & AI e NA \\
\hline \multicolumn{3}{|l|}{ BIGNONIACEAE } \\
\hline Tabebuia serratifolia (Vanl) G. Nicholson & Ipê & AI \\
\hline \multicolumn{3}{|l|}{ BORAGINACEAE } \\
\hline Cordia sp1. & Gagaúba & NA \\
\hline Cordia sp2. & Louro Frei Jorge & NA \\
\hline \multicolumn{3}{|l|}{ BURCERACEAE } \\
\hline Protium heptaphyllum (Aubl.) Marchand & Amescla & AI e NA \\
\hline \multicolumn{3}{|l|}{ CANNABACEAE } \\
\hline Celtis sp. & Cabrinha & AI e NA \\
\hline \multicolumn{3}{|l|}{ CECROPIACEAE } \\
\hline Cecropia palmata Willd. & Imbaúba & AI e NA \\
\hline \multicolumn{3}{|l|}{ CHRYSOBALANACEAE } \\
\hline Hirtella americana $\mathrm{L}$. & Quina-Quina & NA \\
\hline Licania rigida Benth. & Oiticica & AI e NA \\
\hline \multicolumn{3}{|l|}{ CLUSICACEAE } \\
\hline Vismia guianensis (Aubl) Pers. & Pau Sangue & AN \\
\hline \multicolumn{3}{|l|}{ ERYTROXYLACEAE } \\
\hline Erythroxylum simonis Plowman & Guarda Orvalho & AI e AN \\
\hline \multicolumn{3}{|l|}{ EUPHORBIACEAE } \\
\hline Pera glabrata (Schott) Baill. & Cocão & AI e AN \\
\hline sp. & Tamanqueiro & AN \\
\hline
\end{tabular}


Tabela 1. Continuação.

\begin{tabular}{|c|c|c|}
\hline Família/Espécie & Nome Vulgar & Ambiente \\
\hline \multicolumn{3}{|l|}{ FABACEAE } \\
\hline Albizia polycephala (Benth.) Killip ex Record & Vassourinha & AI e AN \\
\hline Albizia sp. & Bordão de Velho & AI e AN \\
\hline Bowdichia virgilioides Kunth & Sucupira & AN \\
\hline Bowdichia sp1. & Sucupira Cancha & $\mathrm{AI}$ \\
\hline Himenaea courbaril L. & Jatobá & AN \\
\hline Inga ingoides (Rich) Will. & Ingá & AI e AN \\
\hline Leuchocarpus araripensis Benth. & Piaca & AN \\
\hline Machaerium aculeata (Raddi) DC. & Espinheiro Rei & AI e AN \\
\hline Pterogyne nitens Tul. & Madeira Nova & AI \\
\hline Senna georgica H.S. Irwin \& Barneby & Lava Prato & AN \\
\hline Piptadenia viridiflora (Kunth) Benth. & Amorosa & AN \\
\hline \multicolumn{3}{|l|}{ LAMIACEAE } \\
\hline Vitex rufescens A. Juss. & Mama Cachorro & AN \\
\hline \multicolumn{3}{|l|}{ LAURACEAE } \\
\hline Ocotea glomerata (Nees) Mez. & Louro Preto & AI e AN \\
\hline Ocotea sp. & Louro Canela & AI e AN \\
\hline \multicolumn{3}{|l|}{ LECYTHIDACEAE } \\
\hline Eschweilera ovata (Cambess.) Miers. & Imbiriba & AI e AN \\
\hline \multicolumn{3}{|l|}{ MALPGHIACEAE } \\
\hline Byrsonima sericea DC. & Muricí & $\mathrm{AI}$ e AN \\
\hline \multicolumn{3}{|l|}{ MALVACEAE } \\
\hline Luehea olivaricata Mart. & Pereiro & AN \\
\hline \multicolumn{3}{|l|}{ MELASTOMATACEAE } \\
\hline Miconia hypoleuca Cogn. & Cinzeiro & AI e AN \\
\hline Tibouchina candolleana DC. Cogn. & Guachumbi & AI e AN \\
\hline sp1. & Manacá & AN \\
\hline \multicolumn{3}{|l|}{ MORACEAE } \\
\hline Artocarpus heterophyllus Lam. & Jaca & AI e AN \\
\hline Brosimum guianense (Aubl.) Huber & Quiri & AI e AN \\
\hline Sorocea ilicifoilia Miq. & Burra Leitera & AI e AN \\
\hline sp1. & Tatajuba & AI e AN \\
\hline \multicolumn{3}{|l|}{ MYRTACEAE } \\
\hline Campomanesia synchrona O. Berg. & Guabiraba & AI e AN \\
\hline Myrcia silvatica Barb. Rodr. & Cuipuna & $\mathrm{AI}$ e $\mathrm{AN}$ \\
\hline Myrcia sp1. & Cuipuna Branca & AI e AN \\
\hline Myrcia sp2. & Cuipuna Rosa & AI \\
\hline Myrcia sp3. & Guabiraba de Soim & $\mathrm{AI}$ e AN \\
\hline Myrciaria cauliflora (Mart.) O. Berg & Jaboticaba & $\mathrm{AI}$ e AN \\
\hline sp1. & Canela de Veado & AN \\
\hline
\end{tabular}


Tabela 1. Continuação.

\begin{tabular}{|c|c|c|}
\hline Família/Espécie & Nome Vulgar & Ambiente \\
\hline \multicolumn{3}{|l|}{ NYCTAGINACEAE } \\
\hline Guapira opposita (Vell.) Reitz. & João Mole & $\mathrm{AI}$ e $\mathrm{AN}$ \\
\hline \multicolumn{3}{|l|}{ PIPPERACEAE } \\
\hline Piper aduncun $\mathrm{L}$. & Pimenta Dada & $\mathrm{AI}$ e $\mathrm{AN}$ \\
\hline \multicolumn{3}{|l|}{ POLYGONACEAE } \\
\hline Coccolobo sp. & Crauassú & AN \\
\hline \multicolumn{3}{|l|}{ RUBIACEAE } \\
\hline Coffea arabica $\mathrm{L}$. & Café & AN \\
\hline Guettarda sericea Müll. Arg. & Pau Jitó & $\mathrm{AI}$ e $\mathrm{AN}$ \\
\hline \multicolumn{3}{|l|}{ SALICACEAE } \\
\hline Casearia hirsuta SW. & Café Bravo & $\mathrm{AI}$ e $\mathrm{AN}$ \\
\hline Casearia sylvestris SW. & Carniceiro & $\mathrm{AI}$ e $\mathrm{AN}$ \\
\hline \multicolumn{3}{|l|}{ SAPINDACEAE } \\
\hline Allophylus laevigatus Radlk. & Estraladeira & $\mathrm{AI}$ e $\mathrm{AN}$ \\
\hline Cupania revoluta Rolfe & Caboatã de Rego & $\mathrm{AI}$ e $\mathrm{AN}$ \\
\hline Cupania sp1. & Caboatã Liso & $\mathrm{AI}$ e $\mathrm{AN}$ \\
\hline Talisia esculenta (A. St.Hil) Radlk. & Pitomba & $\mathrm{AI}$ e $\mathrm{AN}$ \\
\hline Talisia sp1. & Pitomba Branca & $\mathrm{AI}$ e $\mathrm{AN}$ \\
\hline \multicolumn{3}{|l|}{ SAPOTACEAE } \\
\hline Chrysophyllum sp1. & Pororoca & $\mathrm{AI}$ e AN \\
\hline Chrysophyllum sp2. & Laque & AN \\
\hline \multicolumn{3}{|l|}{ SIMAROUBACEAE } \\
\hline Simarouba versicolor A. St. Hil & Praiba & $\mathrm{AI}$ e $\mathrm{AN}$ \\
\hline \multicolumn{3}{|l|}{ SOLANACEAE } \\
\hline Capsicum parvifolium Sendth. & Pimenta de Cutia & AN \\
\hline \multicolumn{3}{|l|}{ TILIACEAE } \\
\hline Apeiba tibourbou Aubl. & Jangada & AN \\
\hline \multicolumn{3}{|l|}{ INDETERMINADA } \\
\hline sp1. & Espinheiro Verde & AN \\
\hline sp2. & Feijão de Bode & AN \\
\hline sp3. & Mariá & AN \\
\hline sp4. & Moranguinho & AN \\
\hline sp5. & Pinheiro Verde & AN \\
\hline sp6. & Pitiá & AN \\
\hline sp7. & Sebo de Carneiro & AN \\
\hline
\end{tabular}

A diversidade e a equabilidade geral foram, respectivamente, 0,24 e 0,06 no AI e 3,42 e 0,79 no AN. A média da diversidade das parcelas foi $0,2788 \pm 0,2893$ no AI e 2,5168 $\pm 0,4076$ no AN. Essa diferença foi altamente significativa $(\mathrm{t}=106,97 ; \mathrm{p}<0,0001)$ (Tab. 2). Além da exclusão de espécies, a grande abundância da exótica em relação às demais, fez com que a equabilidade fosse sensivelmente diminuída refletindo sobre a diversidade do AI. Em área invadida por A. heterophyllus no Parque Nacional da Tijuca, Rio de Janeiro, os valores de diversidade foram semelhantes aos do AI do presente estudo (Abreu \& Rodrigues 2010). Esses valores foram muito inferiores aos de outras áreas de "brejo de altitude" (formação florestal disjunta de Mata Atlântica) (Andrade et al. 2006, Oliveira et al. 2006, Xavier et al. 2009), 

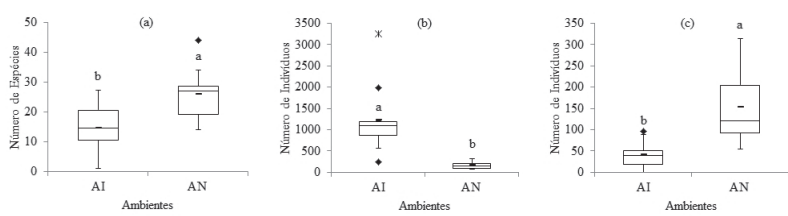

Figura 1. Boxplot do número de espécies (a), número de indivíduos incluindo Artocarpus heterophyllus (b) e número de indivíduos excluindo Artocarpus heterophyllus (c) por ambiente em um fragmento de Mata Atlântica, Areia, PB. Sendo: AI - Ambiente invadido; AN - Ambiente Natural. O box representa 50\% da amostra, os traço a média aritmética, o losango valores isolados e o asterisco valores extremos. Letras iguais não diferem entre si a uma probabilidade $\leq 0,05$, onde (a) e (c) comparados por Tukey e (b) por Kruskal-Wallis.

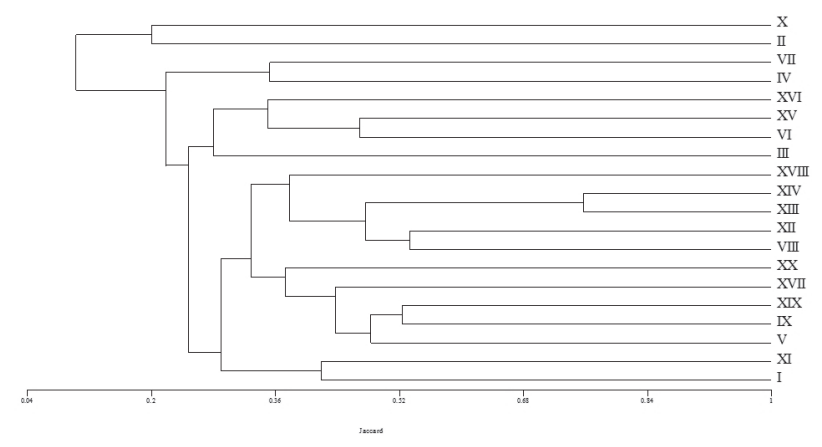

Figura 2. Dendrograma de similaridade florística obtido por uma matriz de presença/ausência, das parcelas amostradas em um fragmento de Mata Atlântica, Areia, PB, utilizando o quociente de Jaccard. Sendo: I-X - parcelas do Ambiente Invadido (AI); XI-XX - parcelas do Ambiente Natural (AN).

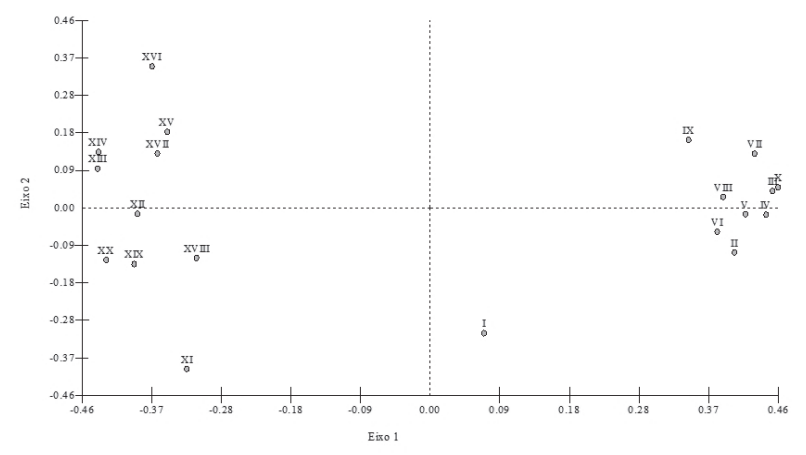

Figura 3. Ordenação por distância de Bray-Curtis das parcelas amostradas em um fragmento de Mata Atlântica, Areia, PB. Sendo: I-X - parcelas do Ambiente Invadido (AI); XI-XX - parcelas do Ambiente Natural (AN).

sendo que os do AN foram semelhantes aos destas áreas, indicando que a espécie afeta de forma incisiva a biodiversidade de sítios invadidos.

As concentrações de Fósforo, Potássio, Sódio, Cálcio, Carbono e Matéria Orgânica, não diferiram significativamente, ou seja, mantiveram-se nas mesmas proporções. Já o pH, e as concentrações de Cálcio+Magnésio, Magnésio, Alumínio e Hidrogênio+Alumínio apresentaram alterações significativas (Tab. 3). O pH teve sua concentração elevada pela presença da espécie, diminuindo a acidez dos solos. O Cálcio+Magnésio também apresentaram alterações significativas, porém, quando analisados separadamente, observou-se que apenas o magnésio difere entre os ambien- tes, neste caso os sítios invadidos tiveram o aumento deste elemento em particular. No caso do Hidrogênio+Alumínio e do alumínio, observou-se que suas alterações foram na diminuição no ambiente invadido.

Provavelmente, as alterações se devem ao aumento significativo na densidade de plantas por área. Conforme Breytenbach (1986), isso implica em modificações drásticas no consumo dos recursos naturais disponíveis. As invasões biológicas podem causar impactos em diversos níveis, como por exemplo, sobre os processos dos ecossistemas, tais como a disponibilidade de nutrientes (Parker et al. 1999). Alguns táxons são capazes de promover alterações nesses processos, como é o caso de espécies de Pinus, que alteram o pH dos solos com consequentes alterações na microfauna e microflora, inviabilizando assim a sobrevivência de espécies de vertebrados e invertebrados (Rapoport 1991).

Segundo a análise de modelagem de nicho, $A$. heterophyllus tem uma área potencial de dispersão/invasão que compreende parte significativa dos domínios de Mata Atlântica (Floresta Ombrófila Densa, Floresta Ombrófila Aberta, Floresta Ombrófila Mista e Floresta Estacional Semidecidual - IBGE 1992), principalmente as regiões mais próximas ao litoral, cujo grau de vulnerabilidade varia de médio a alto. Também se observa uma pequena porção de Cerrado (Savana - IBGE 1992) com susceptibilidade de ocorrência variando de média a baixa (Fig. 4).

A área potencial apontada para A. heterophyllus, está inserida nas áreas brasileiras prioritárias para a conservação da biodiversidade (Hotspots) pela Organização Conservation International (2011). Destaca-se que juntas, Mata Atlântica e Cerrado, possuem mais de 12.000 espécies vegetais endêmicas (Conservation International 2011), que perdem a cada ano espaço para atividades humanas e ainda para espécies exóticas, como a aqui estudada.

Frisa-se que apesar de apresentar potencial de ocorrência nessas regiões, não são em todas as áreas que a espécie apresenta o mesmo comportamento. Em algumas áreas, a espécie está representada primordialmente por indivíduos adultos, sem indícios de regeneração natural expressiva (L. Freitas, comun. pess.). Até o momento, não está claro quais fatores atuariam para diferenciar o comportamento destas populações, cabendo estudos nesse sentido.

Conclui-se que a espécie $A$. heterophyllus causa significativas alterações na riqueza, diversidade e solos dos sítios invadidos estudados. A despeito da importância da espécie como recurso forrageiro para a fauna nativa, e como alimento para o homem, A. heterophyllus deve ser considerada uma importante ameaça à vegetação nativa, tornando-se necessário e urgente ações de manejo e controle da mesma, principalmente em fragmentos de Mata Atlântica onde já se pode observar regenerantes da espécie. 
Tabela 2. Diversidade de Shannon-Weaver $\left(H^{\prime}\right)$ e equabilidade de Pielou (E) das parcelas amostradas em um fragmento de Mata Atlântica, Areia, PB. Sendo: $\mathrm{I}$-X - parcelas do Ambiente Invadido (AI); XI-XX - parcelas do Ambiente Natural (AN).

\begin{tabular}{|c|c|c|}
\hline Amostras & $H^{\prime}$ & E \\
\hline I & 0,886 & 0,327 \\
\hline II & 0 & 0 \\
\hline III & 0,137 & 0,055 \\
\hline IV & 0,158 & 0,062 \\
\hline V & 0,328 & 0,108 \\
\hline VI & 0,544 & 0,176 \\
\hline VII & 0,053 & 0,023 \\
\hline VIII & 0,533 & 0,162 \\
\hline IX & 0,09 & 0,034 \\
\hline $\mathrm{X}$ & 0,059 & 0,037 \\
\hline Média \pm d.p. & $0,279 \pm 0,289$ & $0,098 \pm 0,099$ \\
\hline Geral & 0,24 & 0,06 \\
\hline XI & 2,427 & 0,721 \\
\hline XII & 3,077 & 0,823 \\
\hline XIII & 3,06 & 0,875 \\
\hline XIV & 2,872 & 0,892 \\
\hline XV & 2,355 & 0,732 \\
\hline XVI & 2,332 & 0,823 \\
\hline XVII & 1,728 & 0,655 \\
\hline XVIII & 2,3 & 0,734 \\
\hline XIX & 2,402 & 0,831 \\
\hline $\mathrm{XX}$ & 2,615 & 0,793 \\
\hline Média \pm d.p. & $2,517 \pm 0,408$ & $0,788 \pm 0,075$ \\
\hline Geral & 3,42 & 0,79 \\
\hline Total & 0,866 & 0,198 \\
\hline
\end{tabular}

Tabela 3. Química e fertilidade dos solos dos ambientes estudados. Sendo: AI - ambiente invadido; $\mathrm{AN}$ - ambiente natural. Letras iguais não diferem (ns) entre si a uma probabilidade $\leq 0,05$ segundo o teste Tukey.

\begin{tabular}{lcc}
\hline Elementos & \multicolumn{2}{c}{ Ambientes } \\
\hline \multirow{2}{*}{$\mathrm{pH}$} & $\mathrm{AI}$ & $\mathrm{AN}$ \\
\cline { 2 - 3 } Fósforo & $5,57 \pm 0,33^{\mathrm{a}}$ & $5,01 \pm 0,49^{\mathrm{b}}$ \\
Potássio & $2,63 \pm 0,94^{\mathrm{a}}$ & $2,56 \pm 0,89^{\mathrm{a}}$ \\
Sódio & $83,15 \pm 37,16^{\mathrm{a}}$ & $65,15 \pm 37,85^{\mathrm{a}}$ \\
Calcio+Magnésio & $0,19 \pm 0,09^{\mathrm{a}}$ & $0,16 \pm 0,09^{\mathrm{a}}$ \\
Cálcio & $5,38 \pm 1,74^{\mathrm{a}}$ & $3,02 \pm 1,67^{\mathrm{b}}$ \\
Magnésio & $2,61 \pm 0,56^{\mathrm{a}}$ & $1,96 \pm 1,13^{\mathrm{a}}$ \\
Alumínio & $2,78 \pm 1,62^{\mathrm{a}}$ & $1,07 \pm 0,67^{\mathrm{b}}$ \\
Hidrogênio+Alumínio & $0,07 \pm 0,13^{\mathrm{a}}$ & $0,52 \pm 0,55^{\mathrm{b}}$ \\
Carbono & $5,35 \pm 1,73^{\mathrm{a}}$ & $8 \pm 3,49^{\mathrm{b}}$ \\
Matéria Orgânica ${ }^{\mathrm{n} s}$ & $19,17 \pm 3,44^{\mathrm{a}}$ & $18,95 \pm 5,12^{\mathrm{a}}$ \\
\hline
\end{tabular}

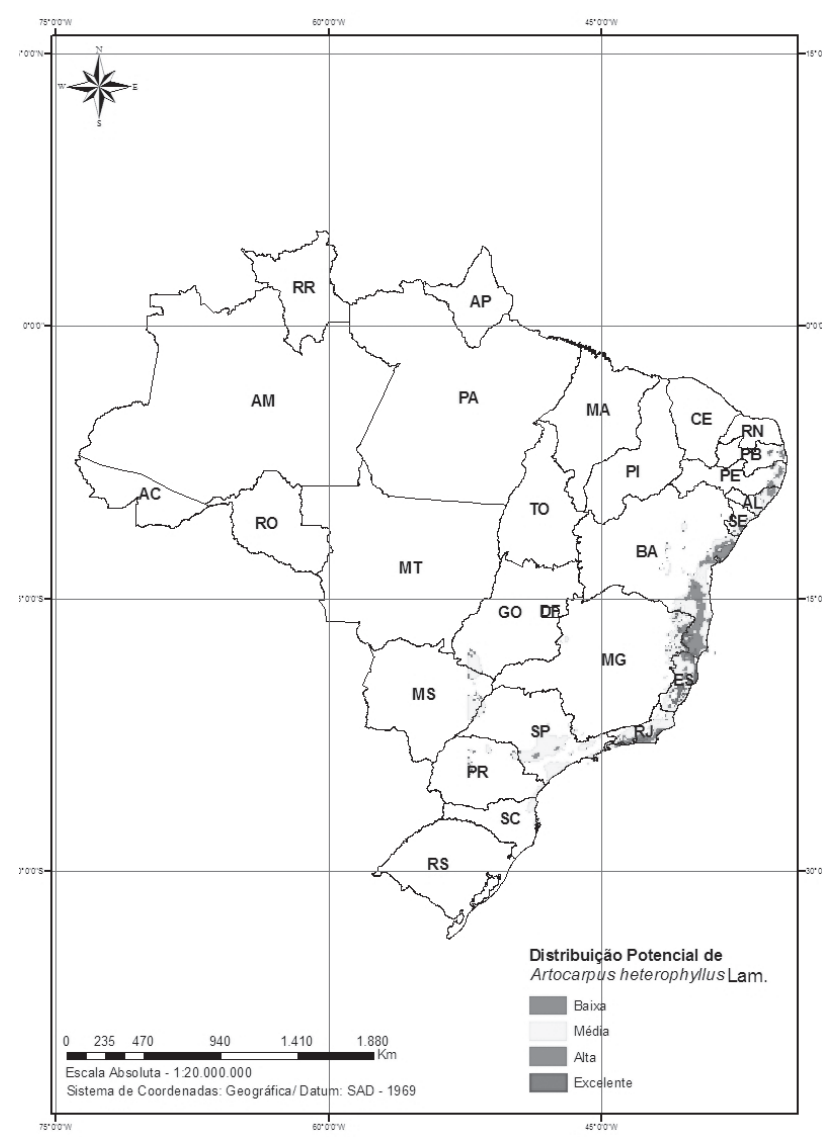

Figura 4. Área potencial de dispersão/invasão de Artocarpus heterophyllus no Brasil, a paritr de modelagem de nicho ecológico utilizado o algoritmo BIOCLIM.

\section{Referências bibliográficas}

Abreu, R.C.R. 2008. Dinâmica de populações da espécie exótica invasora Artocarpus heterophyllus L. (Moraceae) no Parque Nacional da Tijuca - Rio de Janeiro. Dissertação de Mestrado em Botânica, Escola Nacional de Botânica Tropical, Rio de Janeiro.

Abreu, R.C.R. \& Rodrigues, P.J.F.P. 2005. Estrutura de populações de jaqueiras, subsídios para manejo e conservação da Mata Atlântica. In: I Simpósio Brasileiro sobre Espécies Exóticas Invasoras. Brasília.

Abreu, R.C.R. \& Rodrigues, P.J.F.P. 2010. Exotic tree Artocarpus heterophyllus (Moraceae) invades the Brazilian Atlantic Rainforest. Rodriguésia 61(4): 677-688.

Andrade, L.A.; Fabricante, J.R. \& Alves, A.S. 2008. Algaroba (Prosopis juliflora (Sw.) DC.): Impactos sobre a Fitodiversidade e Estratégias de Colonização em Área Invadida na Paraíba, Brasil. Natureza \& Conservação 6(1): 61-67.

Andrade, L.A.; Fabricante, J.R. \& Oliveira, F.X. 2009. Invasão biológica por Prosopis juliflora (Sw.) DC.: impactos sobre a diversidade e a estrutura do componente arbustivo-arbóreo da Caatinga no Estado do Rio Grande do Norte, Brasil. Acta Botanica Brasilica 23(4): 935-943.

Andrade, L.A.; Fabricante, J.R. \& Oliveira, F.X. 2010. Impactos da invasão de Prosopis juliflora (sw.) DC. (Fabaceae) sobre o estrato arbustivo arbóreo em áreas de Caatinga no Estado da Paraíba Brasil. Acta Scientiarum. Biological Sciences 32(3): 240-255.

Andrade, L.A.; Oliveira, F.X.; Nascimento, I.S.; Fabricante, J.R.; Sampaio, E.V.S.B. \& Barbosa, M.R.V. 2006. Analise florística e estrutural de matas ciliares ocorrentes em brejo de altitude, no município de Areia, Paraíba. Revista Brasileira Ciências Agrárias 1(1): 31-40.

APG. 2009. An update of the Angiosperm Phylogeny Group classification for the orders and families of flowering plants: APG III. Botanical Journal of the Linnean Society 161: 105-121. 
Ayres, M.; Ayres, M.J.; Ayres, D.L. \& Santos, S.A. 2007. Bioestat 5.0: aplicações estatísticas nas áreas das Ciências Biomédicas. Belém, Mamirauá/CNPq.

Bandeira, C.M. 1993. Parque Nacional da Tijuca. Markom Books, São Paulo.

Boni, R.; Novelli, F.Z. \& Silva, A.G. 2009. Um alerta para os riscos de bioinvasão de jaqueiras, Artocarpus heterophyllus Lam., na Reserva Biológica Paulo Fraga Rodrigues, antiga Reserva Biológica Duas Bocas, no Espírito Santo, Sudeste do Brasil. Natureza on line 7: 51-55.

Brasil. 1972. Ministério da Agricultura. Levantamento exploratório: reconhecimento de solos do estado da Paraíba. Rio de Janeiro: SUDENE (Boletim Técnico, 15).

Bray, R.J. \& Curtis, J.T. 1957. An ordination of the upland forests communities of southern Winsconsin. Ecological Monography 27: 325-349.

Breytenbach, G.J. 1986. Impacts of alien organisms on terrestrial communities with emphasis on communities of the south-western Cape. Pp. 229-238. In: Macdonald, I.A.W.; Kruger, F.J; Ferrara, A.A. The ecology and management of biological invasions in Southern Africa. Cape Town, Oxford University Press.

Chaves, C.M.; Martins, H.F.; Carauta, J.P.P.; Lanna-Sobrinho, J.P.; Vianna, M.C. \& Silva, S.A.F. 1967. Arboreto Carioca. Centro de Conservação da Natureza, Rio de Janeiro.

Chittibabu, C.V. \& Parthasarathy, N. 2000. Attenuated tree species diversity in humanimpacted tropical evergreen forest sites at Kolli hills, Eastern Ghats, India. Biodiversity and Conservation 9: 1493-1519.

Conservation International. 2011. Biodiversity Hotspots. Disponível em: http:// www.biodiversityhotspots.org/Pages/default.aspx. (Acesso em: 12/02/2011).

Convention On Biological Diversity. 2005. Handbook of the Convention on Biological Diversity Including its Cartagena Protocol on Biosafety. 3rd edition, Montreal, Canada.

CRIA. 2011. Specieslink. Disponível em: http://splink.cria.org.br/. (Acesso em: 02/03/2011).

D’agostino, R.B.; Balanger, A. \& D’agostino Jr, R.B. 1990. A Suggestion for Using Powerful and Informative Tests of Normality. American Statistical Association 44(4): 316-321.

Elith, J.; Graham, C.H.; Anderson, R.P.; Dudik, M.; Ferrier, S.; Guisan, A.; Hijmans, R.J.; Huettmann, F.; Leathwick, J.R.; Lehmann, A.; Li, J.; Lohmann, L.G.; Loiselle, B.A.; Manion, G.; Moritz, C.; Nakamura, M.; Nakazawa, Y.; Overton, J.M.; Peterson, T.A.; Phillips, S.J.; Richardson, K.; Scachetti-Pereira, R.; Schapire, R.E.; Soberon, J.; Williams, S.; Wisz, M.S. \& Zimmermann, N.E. 2006. Novel methods improve prediction of species' distributions from occurrence data. Ecography 29: 129-151.

EMBRAPA. Centro Nacional de Pesquisa de Solos. Manual e Métodos de análise de solo. 2 ed. Rio de Janeiro, EMBRAPA/CPS. 1997.

Ferrão, J.E.M. 1993. A aventura das plantas e os descobrimentos portugueses. Instituto de Investigação Científica Tropical, Lisboa.

Guglieri, A., Caporal, F.J.M. \& Sciamarelli, A. 2009. Modelos de distribuição geográfica de cinco gramíneas invasoras em Mato Grosso do Sul, Brasil. Pp. 834-843. In: Anais do $2^{\circ}$ Simpósio de Geotecnologias no Pantanal, Corumbá, Embrapa Informática Agropecuária/INPE.

Hammer, O.; Harper, D.A. \& Ryan, P.D. 2003. PAST 1.12. Paleontolological Statistics.

Hijmans, R.J.; Guarino, L.; Jarvis, A.; O’brien, R. \& Mathur, P. 2009. DIVAGIS 5.2. International Plant Genetic Resources Institute (IPGRI).

Joly, C.A. et al. 1999. Evolution of the Brasilian phytogeography classification systen: Implication for biodiversity conservation. Ciência e Cultura 51(5/6): 331-348.

IBGE. 1992. Manual técnico da vegetação brasileira. Rio de Janeiro, (Série Manuais Técnicos em Geociências).

Instituto Horus. 2011. Artocarpus heterophyllus. Disponível em: http:// www.institutohorus.org.br/. (Acesso em 02/03/2011).

Kovach, W.L. 2005. MVSP. A MultiVariate Statistical Package for Windows, ver. 3.1. Pentraeth, Wales, Kovach, Computing Services.

Lehmann, E.L. 1997. Testing statistical hypotheses. 2.ed. New York, Springer-Verlag.

Mcknight, T.L. \& Hess, D. 2000. Climate zones and types: the koppen system, physical geography: a landscape appreciation. Upper Saddle River: Prentice Hall.
MMA. 2004. Dados da Mata Atlântica. Disponível em: http://www.mma. gov.br. (Acesso em: 18/10/2004).

Muller-Dombois, D. \& Ellemberg, H. 1974. Aims and methods of vegetation ecology. New York, John Wiley \& Sons.

Novelli, F.Z.; Moreira, R.P.G.; Duca, C. \& Silva, A.G. 2010. O papel da barocoria na estruturação da população da jaqueira, Artocarpus heterophyllus Lam. na Reserva Biológica de Duas Bocas, Cariacica, Espírito Santo. Natureza on line 8(2): 91-94.

Nunes, E.L. 2004. Análise e regeneração de espécies arbóreas em um trecho com plantio de Arthocarpus sp. (jaqueira) na reserva Biológica de Duas Bocas, Cariacica-ES. Trabalho de Conclusão de Curso. Graduação em Ciências Biológicas. Santa Teresa, Escola de Ensino Superior do Educandário Seráfico São Francisco de Assis.

Oliveira, F.X.; Andrade, L.A. \& Félix, L.P. 2006. Comparações florísticas e estruturais entre comunidades de floresta ombrófila aberta com diferentes idades, no município de Areia, Paraíba. Acta Botanica Brasilica 20: 861-873.

Parker, I.M. et al. 1999. Impact: toward a framework for understanding the ecological effects of invaders. Biological Invasions 1: 3-19.

Perdomo, M. \& Magalhães, L.M.S. 2007. Ação alelopática (Artocarpus heterophyllus) em laboratório. Floresta e Ambiente 14(1): 52-55.

Pielou, U.C. 1977. Mathematical diversity. New York, John Wiley.

Rapoport, E.H. 1991. Contaminação por espécies. Ciência Hoje 13(75): 52-57.

Rejmánek M.; Richardson, D.M. \& Pysek, P. 2005. Plant invasions and invasibility of plant communities. Pp. 332-355. In: Van Der Maarel, E. (Ed.), Vegetation ecology, Oxford, Blackwell Publishers.

Ribeiro, M.C.; Metzger, J.P.; Martensen, A.C.; Ponzoni, F.J. \& Hirota, M.M. 2009. The Brazilian Atlantic Forest: how much is left, and how is the remaining forest distributed? Implications for conservation. Biological Conservation 142: 1144-1156.

Richardson, D.M. \& Higgins, S.I. 1998. Pines as invaders in the southern hemisphere. Pp. 450-473. In: Richardson, D.M. (Ed.) Ecology and biogeography of Pinus. Cambridge, Cambridge University Press.

Shannon, C.E. \& Weaver, W. 1949. The Mathematical Theory of Communication. Urbana, University Illinois Press.

Silva, J.M.C.; Tabarelli, M. 2000. Tree species impoverishment and the future flora of the Atlantic forest of northerastern Brazil. Nature 404: 72-74.

Silva, M.C.; Queiroz, J.E.R.; Araujo, K.D.\& Pazera Jr, E. 2006. Condições ambientais da Reserva Ecológica Estadual da Mata Pau Ferro, AreiaPB. Revista Geografia 15(1): 51-63.

SNE. 2002. Mapeamento da Mata Atlântica, seus ecossistemas associados: Paraíba e Rio Grande do Norte. Relatório técnico.

Stehmann, J.R.; Forzza, R.; Salino, A.; Sobral, M.; Costa, D.P.; Kamino, L.H.Y. 2009. Plantas da Floresta Atlântica. Rio de Janeiro, Jardim Botânico do Rio de Janeiro.

TROPICOS. 2011. Tropicos. Disponível em: http://www.tropicos.org/. (Acesso em: 19/03/2011).

Tukey, J.W. 1959. A Quick, Compact, Two-Sample Test to Duckworth.s Speci.cations. Technometrics 1(1): 31

Versfeld, D.B. \& Van Wilgen, B.W. 1986. Impact of woody aliens on ecosystem properties. Pp. 239-246. In: Macdonald, I.A.W.; Kruger, F.J.; Ferrara, A.A. The ecology and management of biological invasions in southern Africa. Cape Town, Oxford University Press.

Xavier, K.R.F.; Andrade, L.A.; Coelho, M.S.E.; Assis, F.N.M. \& Fabricante, J.R.; Impactos do fogo sobre o componente arbustivo-arbóreo de um remanescente de Floresta Ombrófila Aberta, Areia, Estado da Paraíba. Acta Scientiarum. Biological Sciences 31(4): 407-413.

Zar, J. H. 1999. Biostatistical analysis. New Jersey, Prentice-Hall.

Ziller, S.R. \& Galvão, F.A. 2002. Degradação da estepe gramíneolenhosa no Paraná por contaminação biológica de Pinus elliotti e P. taeda. Floresta 32(1): 41-47.

Ziller, S.R. \& Zalba, S. 2007. Propostas de acao para prevencao e controle de especies exoticas invasoras. Natureza \& Conservação 5(2): 8-15. 